\title{
FATIGUE DAMAGE OF RIVET JOINTS IN THE CONDITION OF STABLE CYCLIC LOAD
}

\author{
Volodymyr Hutsaylyuk \\ Lucjan Śnieżek \\ Military University of Technology, Warsaw, Poland \\ Volodymyr Hlado \\ Ternopil National Ivan Pul'uj Technical University, Ternopil, Ukraine
}

\begin{abstract}
The article is devoted, to the attempt of working out of a method for determination of period of exploitation for riveting connections with microdefects on the initial stage and propagation of crack to the moment of appearances to the surface. The tested material used aluminum alloy 2024-T3. Experimental researches are realized on flat specimens from an aluminum alloy 2024-T3 with the row of openings for riveting: without riveting (initial state), with a removal of riveting, and also the specimens connected by riveting at stable amplitude loading (tension, $\sigma=100 \mathrm{MPpa}$ ) at the coefficients of asymmetry of cycle of $R$ : 0,15;0,3 and 0,5. An origin of local damages in the sheets, and also in riveting connections was controlled by the use of vortex-current defectoscope BD 3-71 with a sensor of a converting type PN-12 MDF 01. Researches of mechanisms of fracture and fatigue were realized with the use of electron scanning microscope of REM of 106I. As a result of these research, information was received about the mechanisms of failure, defined a form and a size of initial damage in appearance in a crack, which can be used as a quality parameter for determination of transitional period of exploitation with microdefects. On the basis of the collected experimental results, statistical description of sizes of local damages was made.
\end{abstract}

\section{INTRODUCTION}

Riveting connections are widely used in different industries of machine building and transportation designs. Although, by not looking at the wide implementation of welding connections, there do exist constructions in which part of over $80 \%$ make up riveting joints. To such constructions belong planes from civil and military aviation that assigned to perform different tasks during their exploitation [1].

Exploitation loads lead to natural fatigue processes and aging of material, structural damages and defect accumulation, as on micro and on macro levels.

Especially vulnerable elements during that, without reservations are connection places, which at the same time found under influence of internal factors from side of microstructure and external loads of exploitation [2].

Therefore, to evaluate factual state workability of the construction as a whole, or directly given element, there is a need of considering a connection as a complex, paying special attention on its component elements [3-5]. When adhering to assembly and exploitation norms, natural fatigue processes as a rule in majority exhibit themselves in aluminum sheets, especially in that, which has a hole drilled for the head of a rivet. 
The problem consists in that; damage begins to accumulate under the rivet's head, in the field enclosed for visual control (Fig.1). Also, at the beginning stage of crack propagation, consequently laminating (plating) of the sheet surface and creation of remaining pressing stresses, during process of riveting crack grows in the body of the sheet without appearing to the surface. Damage in join becomes known only after crack appeares to the surface and reaches the size accessible for visual control. At the same time crack propagation into depth of the sheet is practically uncontrollable and may even reach up to $70 \%$ of the sheet thickness. Since usually joint is created by the row of rivets, then moment of cracks creation on the surface at the few rivets might be critical for construction durability, since cross section is weakened due to crack propagation into depth of the sheet. Therefore, consideration of initiation moment and primary crack propagation is an important part of prediction of remaining resource of joint and entire construction.

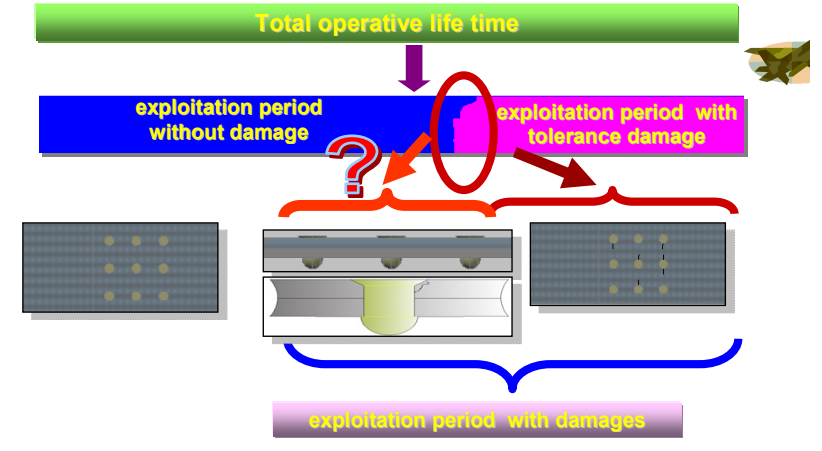

a

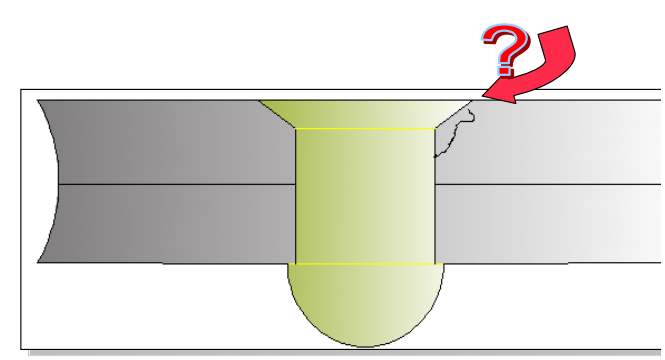

b

Fig. 1 Schema of fatigue defects accumulations during exploitation term (a) and propagation of a primary short crack (b)

A not less important task is a determination of initial fatigue defect in the form of a short crack, which initiates under the head of a rivet in main material of aluminum sheet that is found under overlay and obstructed for visual observation. In this case a problem consist in necessity of use corresponding methods non-destructive control which allow with enough precision determine existence of a damage and to control its parameters.

It is understandable, that from the moment of initiation of short crack (accumulation of critical mass of damages) to the moment of crack appearance on surface all main fracture processes are being completed on micro level. Realization of this or other type of fracture, their consistence in a stage of micro crack formation with sharply defined characteristics of front and direction of propagation onward would maximum influence on remaining resource of joint and lifetime of construction as a whole.

Because of that the goal of this work was an attempt to work out methods for determination of initial damage of material, which could be accepted as primary for defining a period of exploitation with damage up to the moment of its visualisation. Next, not less important goal was a research of micro mechanisms of fracture, which realized on the initiation stage, propagation of crack on the forming stage of macro crack and transition in to stage of final failure.

\section{TEST PROCEDURE AND DISSCUSION}

In the case of research material used aluminum alloy 2023-T3 which widely used for external skin for wings and fuselage of modern planes. Chemical composition and mechanical properties of researched material are shown in Table 1. 
Table 1 [3]

\begin{tabular}{|c|c|c|c|c|c|}
\hline \multicolumn{7}{|c|}{ Chemical structure (\%): } \\
\hline \multirow{3}{*}{$2024-T 3$} & $\mathrm{Cu}$ & $\mathrm{Mg}$ & $\mathrm{Mn}$ & $\mathrm{Fe}$ & $\mathrm{Zn}$ \\
\cline { 2 - 6 } & $3,82-4,04$ & $1,75-1,77$ & 0,56 & $0,08-0,18$ & 0,16 \\
\cline { 2 - 5 } & \multicolumn{2}{|c|}{ Mechanical properties in direct roller (LT) } \\
\cline { 2 - 5 } & $\mathrm{R}_{\mathrm{m}}, \mathrm{MPa}$ & \multicolumn{3}{c|}{$\mathrm{R}_{02}, \mathrm{MPa}$} & $\mathrm{A}, \%$ \\
\cline { 2 - 5 } & $459-466$ & $339-345$ & \multicolumn{2}{c|}{$21,5-24,7$} \\
\hline
\end{tabular}

The specimens for testing were made from sheet and were cut from double-sided lamellar aluminum in direction of rolling. For testing were used three types of unified specimens (Fig. 2): plates $80 \times 250 \mathrm{MM}, 80 \times 420 \mathrm{~mm}$ and "oar shaped" specimens for tensile testing. The holes for rivets were manufactured in such way to ensure that heads of rivets would be with maximum fit to plane of the plate and crossing onto borders of head of plate was minimal. Shorter plates were joined by three rows of rivets, each row had three rivers. Riveting was realized by a press with forming a half a sphere closing head. Plates overlay estimated $40 \mathrm{~mm}$. The longer plates were manufactured with holes for body of a rivet and also for the head. A part of them was joined by rivets in the central part, identically to previous specimens. Prior to testing rivets were removed

Research was realized on testing machine «Instron» (Fig. 3) at cyclical load of stable amplitude $\Delta \sigma=100 \mathrm{MPa}$, at frequence of $15 \mathrm{~Hz}$ and coefficient of asymmetry $\mathrm{R}=0.15,0.3,0.5$.
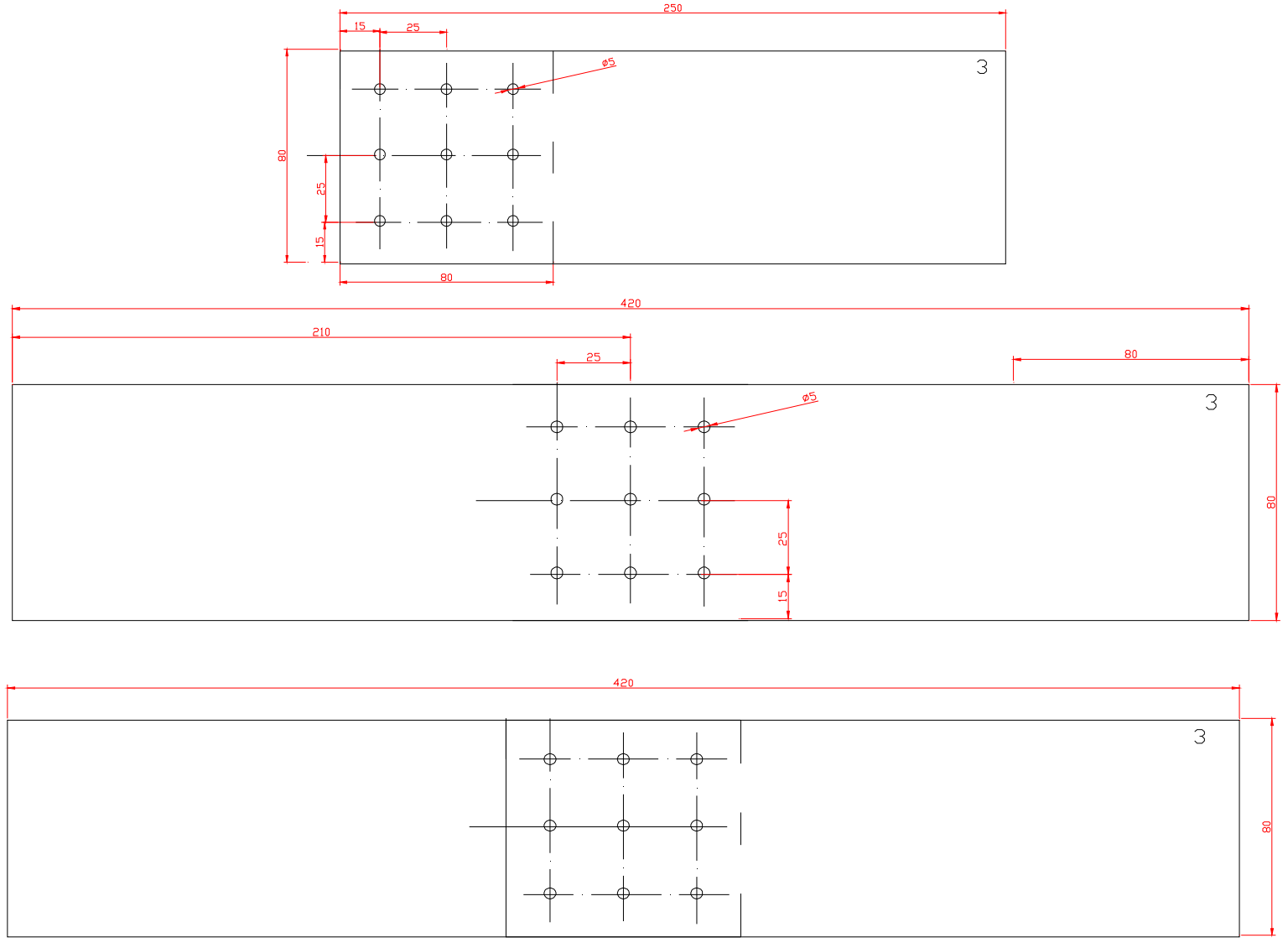

Fig. 2. Specimens for testing 
The testing program anticipated testing at different asymmetry cycles of specimens with holes for body of rivet (underlay) and head (overlay) in virgin state and after prior removal of rivets. First types of specimens were researched in assembled state in all cases.

During testing a control of holes conditions was performed visually and by use of optical microscope at $25 \mathrm{x}$ magnification.

For the control of material damage was used vortex-current defectoscope BD 3-71 with a sensor of a converting type PN-12 MDF 01. The main parameters of the device are listed in Fig.4.

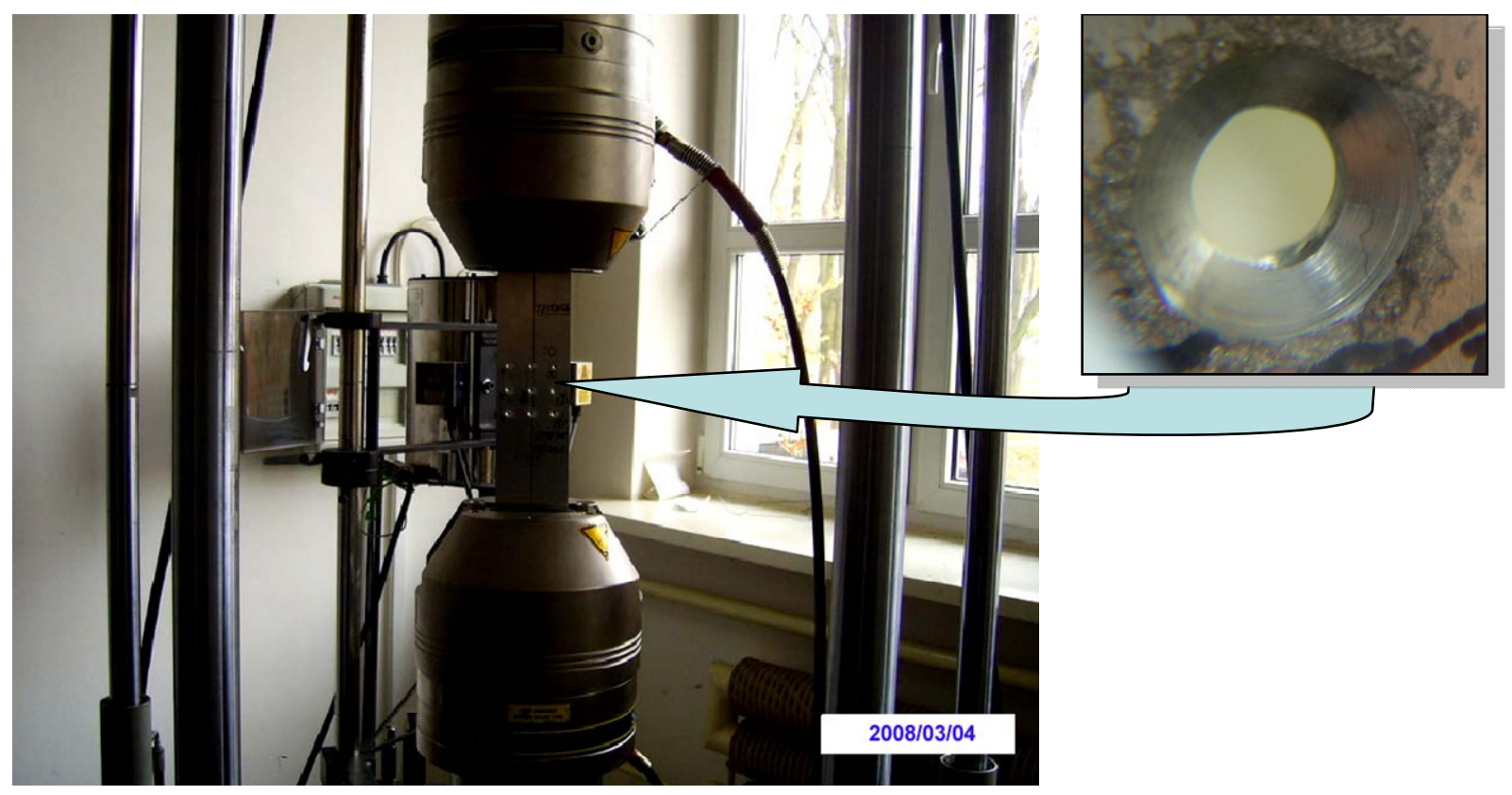

Fig. 3 Testing machine

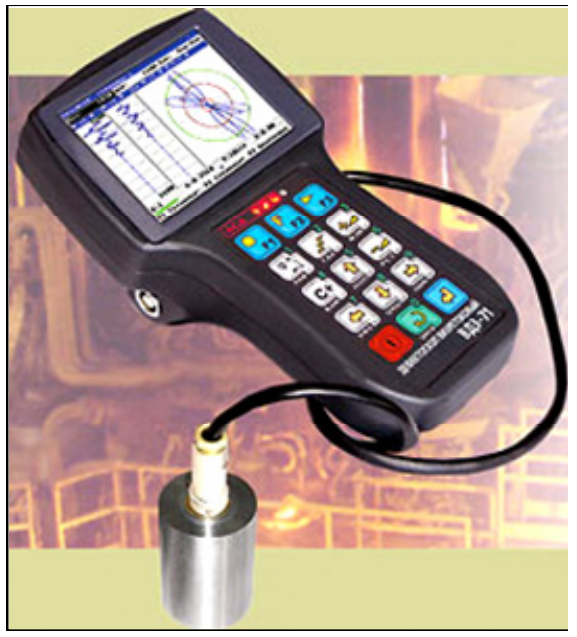

Major technical parameters:

\begin{tabular}{l|l}
\hline Range of working frequencies & $\begin{array}{l}\text { from } 500 \mathrm{~Hz} \text { to } \\
2 \mathrm{MHz}\end{array}$ \\
\hline Terminal volts generator & from 0,5 to $8 \mathrm{~V}$ \\
\hline $\begin{array}{l}\text { Range adjusting multiplication } \\
\text { factor }\end{array}$ & $52 \mathrm{Db}$ \\
\hline Range scaling signal & to $1: 100$ \\
\hline $\begin{array}{l}\text { Frequency discretization } \\
\text { (frequency selection) }\end{array}$ & to 3000 sel./s \\
\hline
\end{tabular}

SENSOR OF TYPE OF PN-12 MDF 01

Fig. 4 Vortex-current defectoscope BD 3-71

Prior calibration of a device was completed by use of master sample with depth from $0,15 \mathrm{~mm}$ до $2 \mathrm{~mm}$. Since sensor is being used in the protective shield, then amplifying coefficient was in 
limits of $8 \mathrm{~V}$, a surveying frequency was tuned in automatically and composed 250 selections per second. To give a warning about growth of defects, limits on values of defects were set, according to the master sample. When signal exceeded set limit (crossing with loop lone of a bordering circle on the complex surface of a screen) additionally light emitting diode LED would light up and alarm would sound. All measurements took place from left to right, from to bottom in a direction perpendicular to predicted crack. For the specimens with holes under the head of a rivet (overlay) from the side of conical hole; for riveting joint from the side of the rivet heads. The result of each measurement was preliminary analyzed on a screen of a defectoscope, and then was stored into the memory of a device for the further analysis in an environment of a computer program BD371_1_11.

Fig. 5 shows the screen shot of the program. The left side of the picture shows a signal in time over two channels and on the right side a complex plain. Hole is recreated in form of two peaks in time (crossing over beaches), and in the complex plane respectively as a loop.

Presence of damages causes deformation of a loop form in the complex plane and bending of the one of peeks on the graph of dependency signal from time.

In accordance with calibration of defectoscope as an initial damage was accepted a crack width of which was $0.1 \mathrm{~mm}$ and depth $0.4 \mathrm{~mm}$.

All measurements were conducted with use of protective shield for the body of a sensor, since on the account of friction; working part of a sensor wears out.

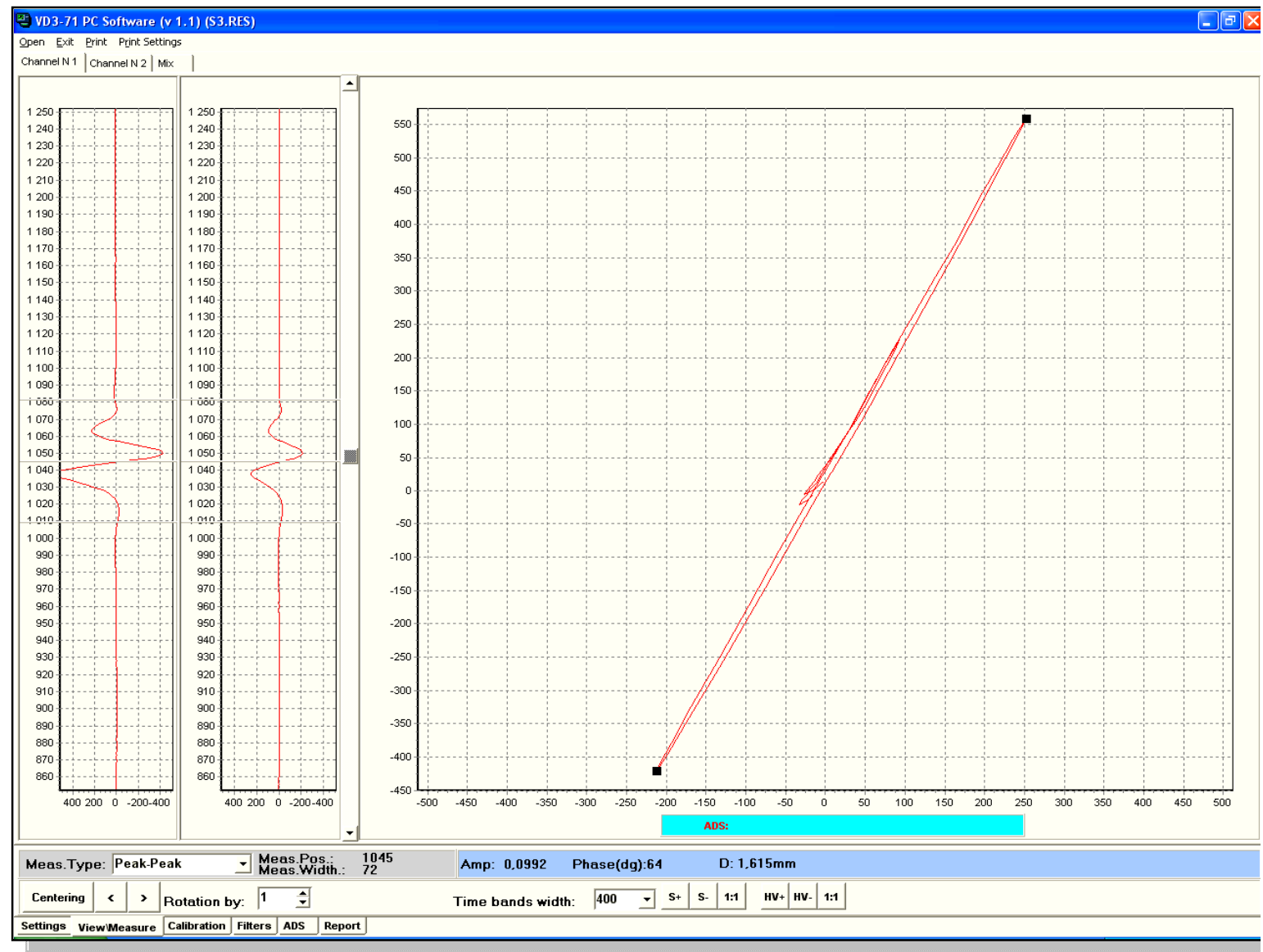

Fig. 5 Screen shot of BD371_1_11program

The measurement of the value of a damage conducted by way of selecting of a peak on a graph by a cursor. Result of each measurement can be printed in the form of a text document (see Fig. 6). The document contain all numerical data and graphical reflection of a signal, however a set limits during measurement in complex plane on Fig. $6 \mathrm{~b}$ on the print are not shown. 


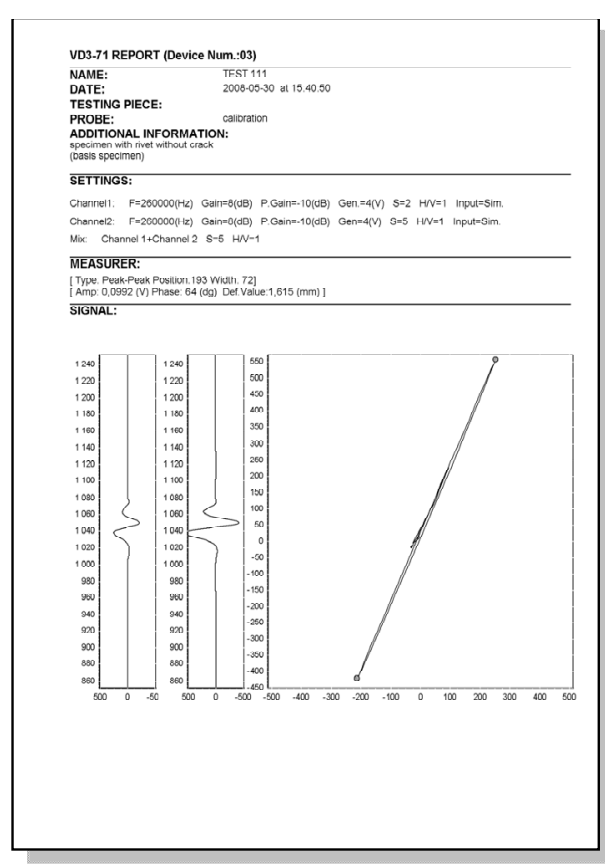

a

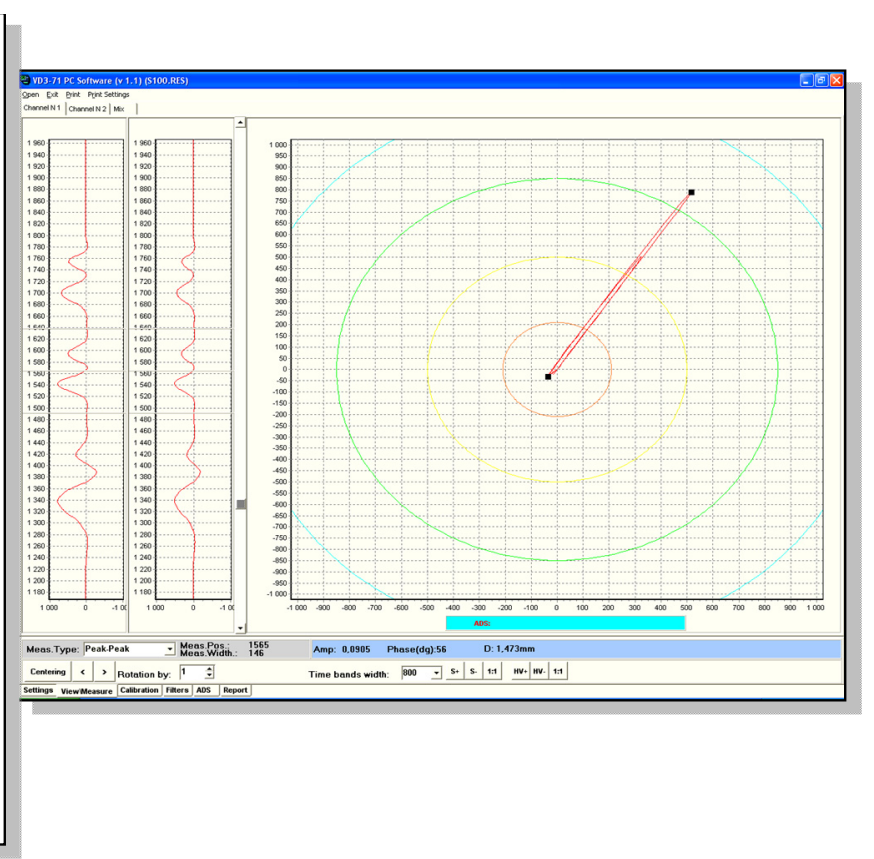

$\mathrm{b}$

Fig. 6 Sample of a report in text document print out (a) and screen shot of a window with set limits (b)

As a result of conducted testing significant quantity of information was obtained which at this time is being worked on. Preliminary results testifying that defectoscope allows to determine presence of damage in the stage of initiation, or when it is not visible yet by the naked eye. After discovery of a defects with a help of defectoscope specimen was removed and surveyed under the optical microscope Neofot 2 under $120 \times$ magnification. On a conical surface of the hole for the head of a rivet a crack was discovered with a length of $500 \mathrm{mkm}$ and width $30 \mathrm{mkm}$, situated under the angle of $45^{\circ}$ to the axis of the hole.

Depending on asymmetry of a cycle and type of tested specimen (overlay), ( underlay) initial defect appeared after $5 \times 10^{4}-10^{5}$ cycles, which made up $0,2-0,35$ of general quantity of cycles to defects.

In the case of underlay defects were registered later and as a rule were located on the inside of the hole, close to the specimen surface.

For the specimen with riveting joints appearance of defect responded to quantity of cycles for overlay. Also it needs to be noted that in all tested specimens destruction was occurring by the account of overlay failure.

Therefore, fractography analysis at first was conducted on broken surface of overlay. At that stage of research surface of a hole in underlay was analyzed only on surface for comparison of a mechanical state of damage on a surface after mechanical treatment(Fig. 7).

For a different asymmetry of a cycle characteristically is different front of a fatigue crack, short and wide in case of $\mathrm{R}=0,15$ and more elongated, closely similar to half-elliptical in the next two (Fig. $7 b, c)$. In the all cases fatigue propagation of a crack was happening in the thickness of a material, practically without surfacing. This illustrates importance of revealing of crack propagation prior to surfacing, because after appearance on the surface it becomes a case of finishing stage of failure and momentary propagation of a crack. On a Fig. 7a the front of a crack has a ripped form, more closely similar to elliptical quadrant. Typical pictures of a surface inside the hole after mechanical treatment are shown on a Fig. 7d. With magnification on the surface clearly are visible local mechanical damages which might be initiators for cracks. Especially vulnerable place is a transition between lamellar and base material. In the case of a hole additionally imposing deformation of a material on the border during riveting which leads to pressing out of metal, Fig. 8 . 

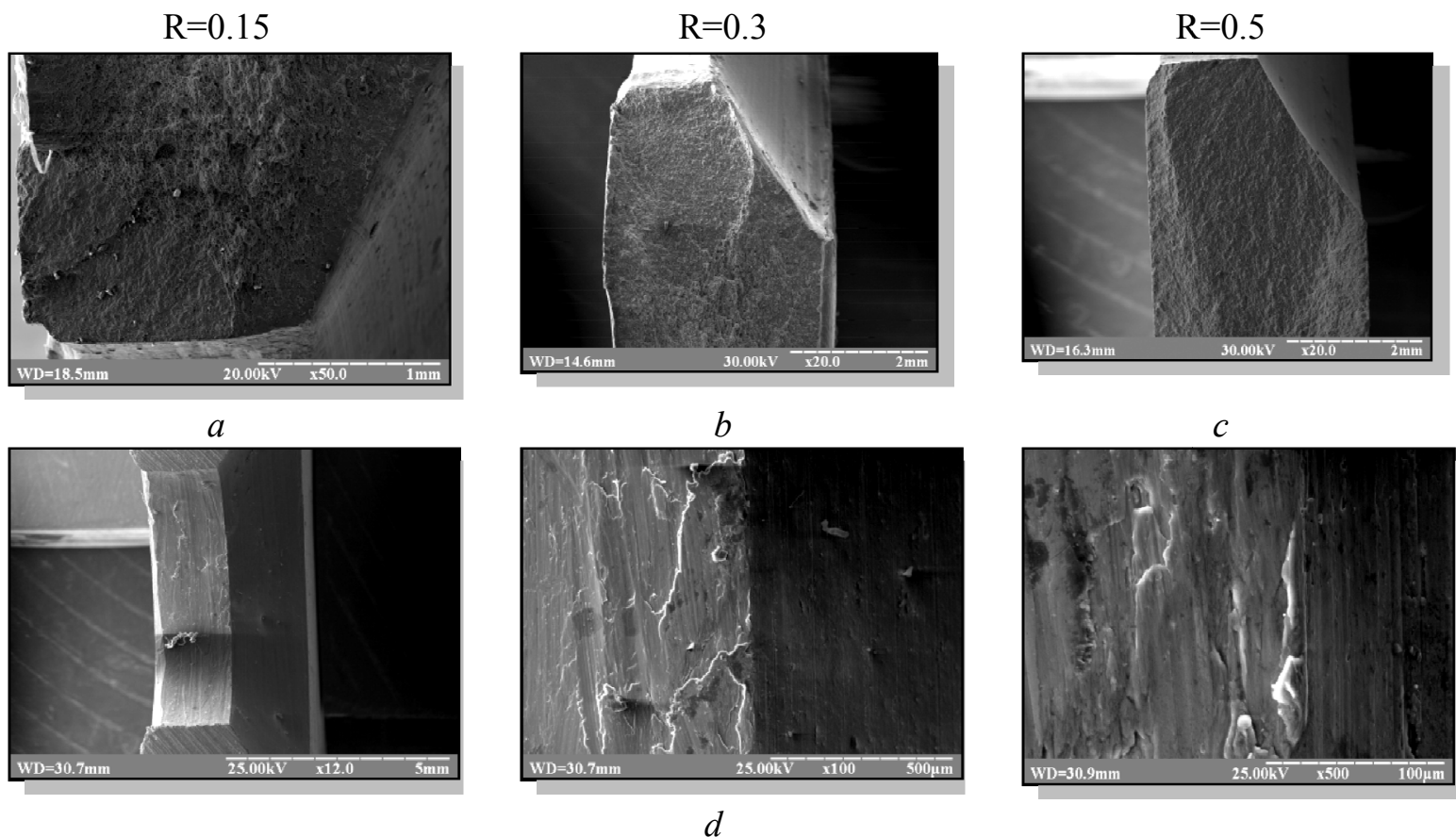

Fig. 7 Source crack initiation on surface failure and area propagation crack in zone near source crack initiation (sem microscope picture)

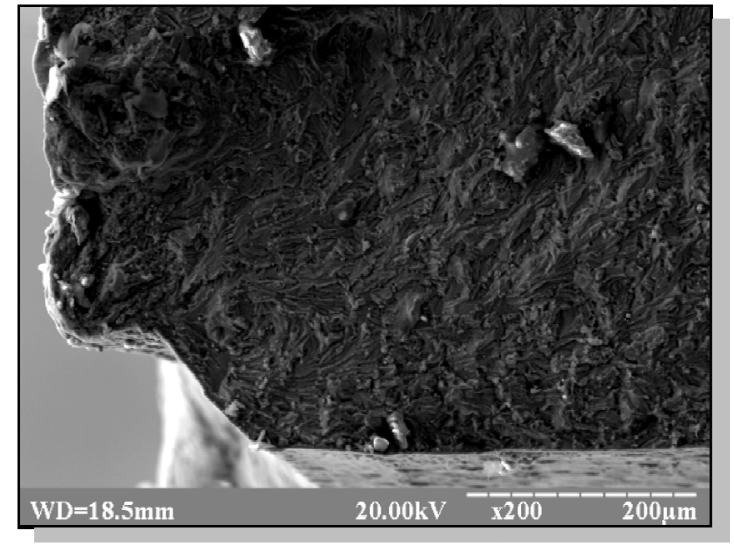

a

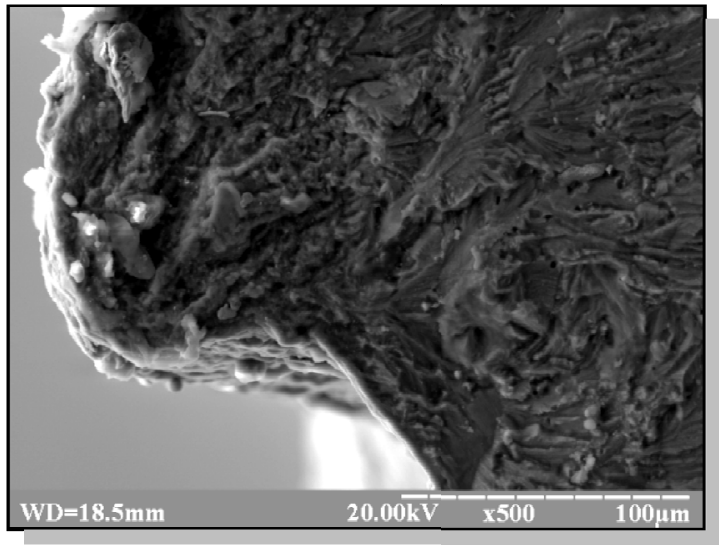

b

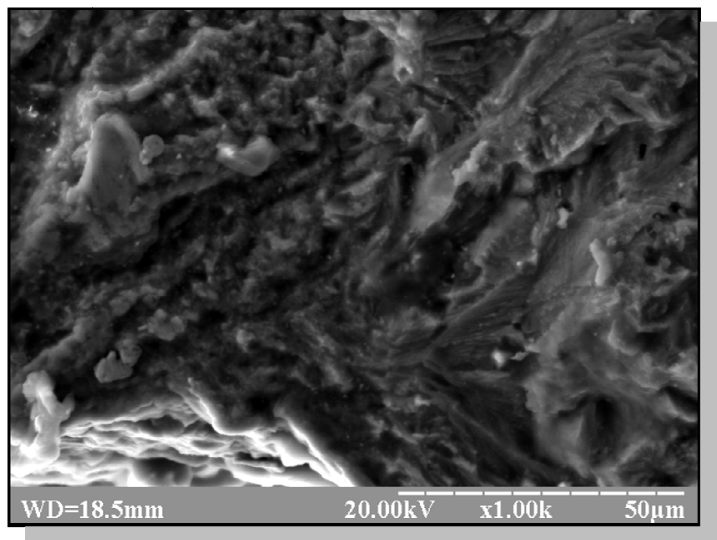

$\mathrm{C}$

Fig.8 Fatigue mechanisms of crack propagation 
On the surface of a fracture clearly visible deep valleys on the bottom of which secondary micro cracks are visible. Generally they are concentrated at the bottom of the pressed out material, facets are quite massive, and respond to size of structural composition, such as grains or sub-grains and created as a result of sliding on corresponding crystal- graphical planes, also visible pits that remain in subsequence to destruction of inter-metal inclusions. Direction of combs is parallel to direction of fatigue crack propagation. On Fig.8a it might be able to distinguish two different types of facets, more crumbled by the surface and a bit elongated towards center of the sheet, which assembly into brook pattern.Obviously this is explained by different level of hardness of surface layer of lamellar, which fails more brittle and base material. Separation of those layers acts as main source of crack initiation, inception of which is stimulated additionally by mechanical damages when making a hole and external loads. On some facets of main material fatigue furrows are observed, which appeared as a result of sliding of crystal-graphical planes. Already at this stage it can be noted the beginning arrangement of facets, that gives a basis to suppose that on this stage crack propagates towards surface of specimen, tunnelling lamellar and moving towards surface of the hole. Only in the next stage crack front would be formed which would define quadrant or half-elliptic macrocrack. General mechanism of fracture realized as consequences of beginning and propagation of cracks on planes of sliding by the way of movement under $45^{\circ}$ angle in regards to axes of stress.

Next character point was a field inside of propagation of fatigue macrocrack, with a certain distance from the layer of lamellar into depth of specimen (Fig. 9)

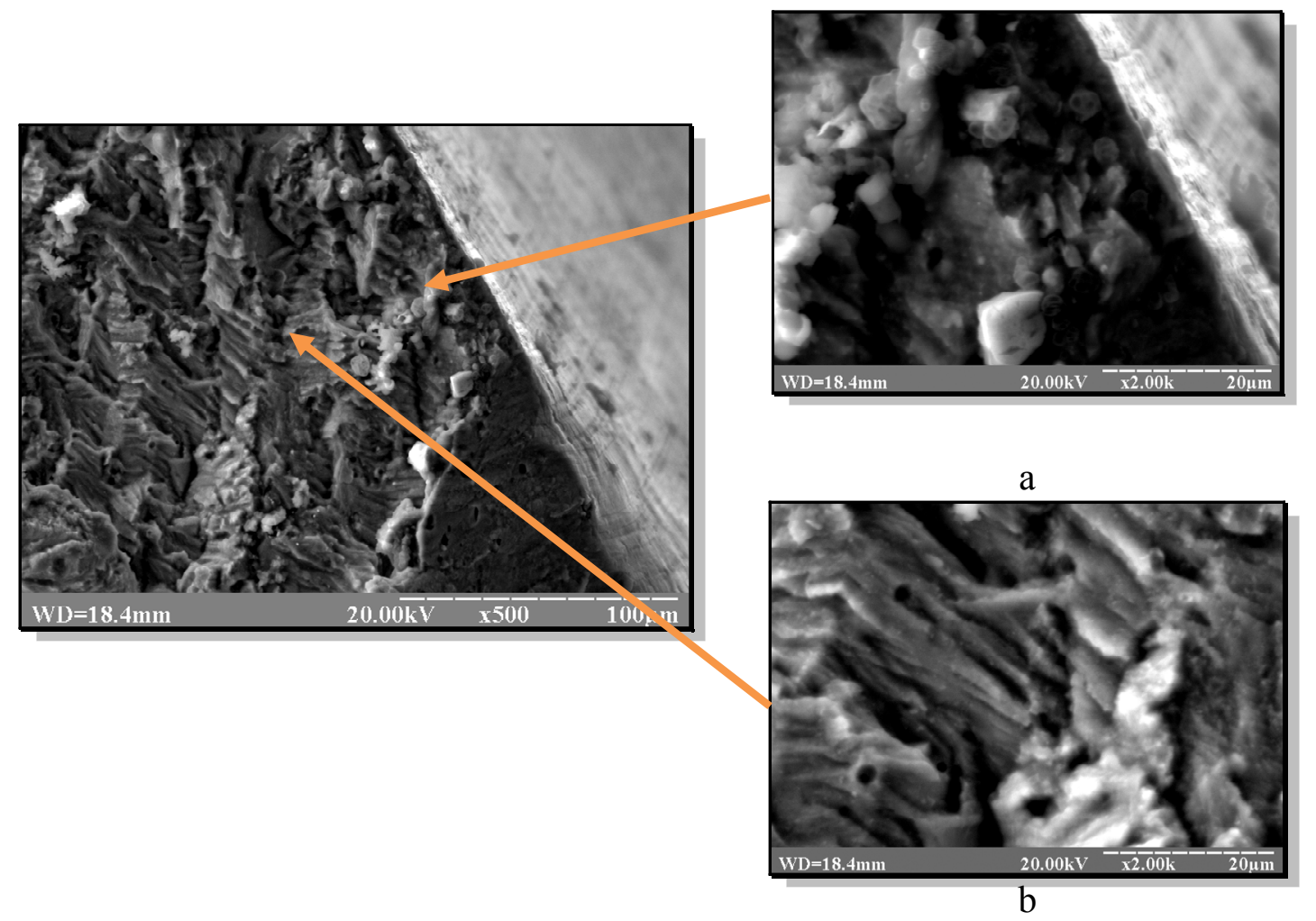

Fig.9 Fatigue mechanisms of crack propagation

The state of surface of fracture was analyzed in two points: one of which is located on the surface of a base material and second on a surface of destruction of lamellar. Fracture of lamellar is characterized by great amount of small facets (Fig. 9a), which have rounded edges and remotely look like spherical objects, secondary cracks and in deepening small pits appear to be present. A general picture of damages in this layer is unclear and might be explained by additional failure of 
elements on the surface in a process of cyclical load during contact with surface. Brittleness of considered layer in a process of loading causes mutual smoothing, cleavage of facets on a surface in the process of failure.

During analysis of a comb in the field of base material, was noted that on the surface of edges clearly visible small round pits, edges are covered with small relief combs which are located from all sides of facet. Such picture can be interpreted as a result of action of complex mechanism of fracture during which branching is occurring of initial crack and initial crack grows simultaneously in all directions of facet edges. So, based on that, it may be assumed that micro-fracture and propagation of macrocracks under cyclical load is happens by the account of whole system of microstructural short cracks that connect into single net.

On the stage of stable propagation of fatigue crack clearly defined fatigue furrows are formed, most character fragments of which are presented on Fig.10. Fatigue furrows located on facets, size of which corresponds to size of alloy structure elements. The orientation and levels of some facets changes from grain to grain. Presence of massive inclusions causes local ductile fracture due to mechanism of pores growth. By comparing small growth of fatigue crack a step of fatigue furrows is smaller (Fig. 10a,b). With an increase in speed of growing of a fatigue crack, step of a furrows increases and increases quantity of secondary cracks (Fig. 10c,d)

It is necessary to note that due to complex stress state in cyclical plastic zone prior to tip of a crack, the direction of lines of fatigue striations may be essentially differ in neighbouring grains, since fatigue crack locally propagates in a direction with minimal energy of fracture. (Fig. 10a,b)

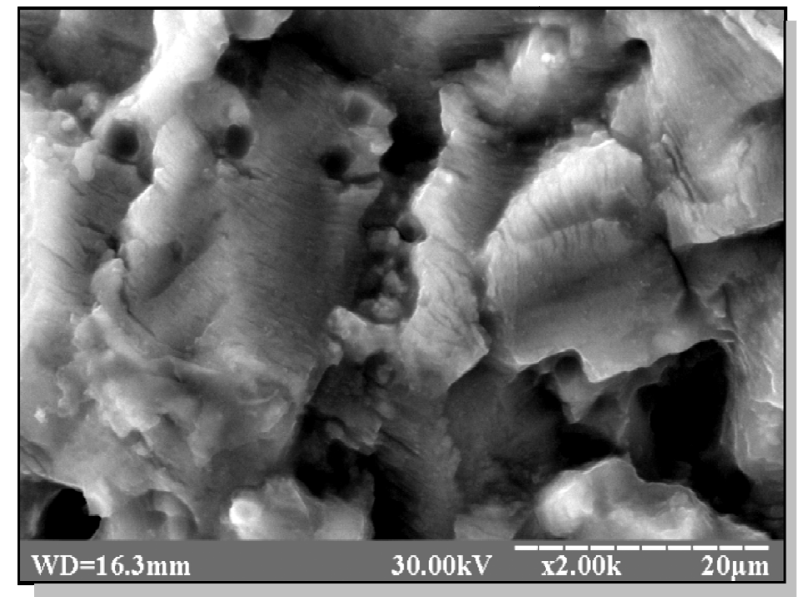

a

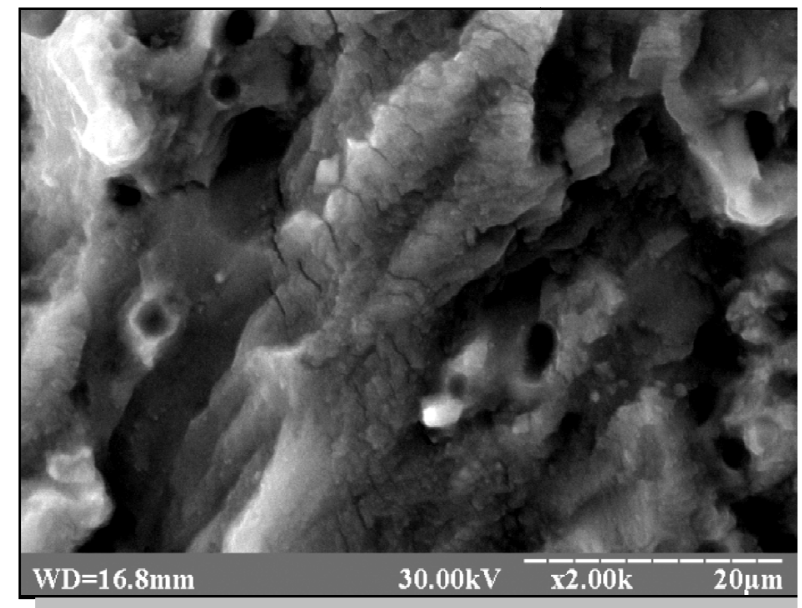

c

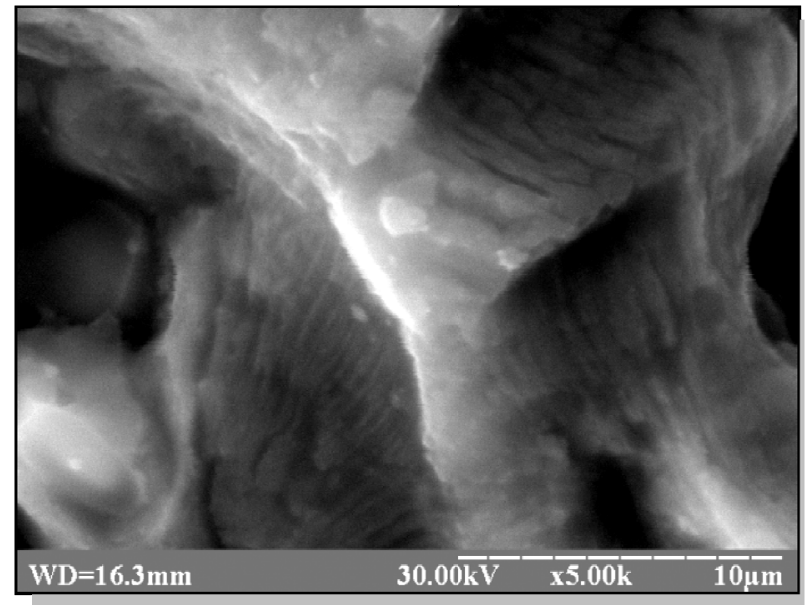

$\mathrm{b}$

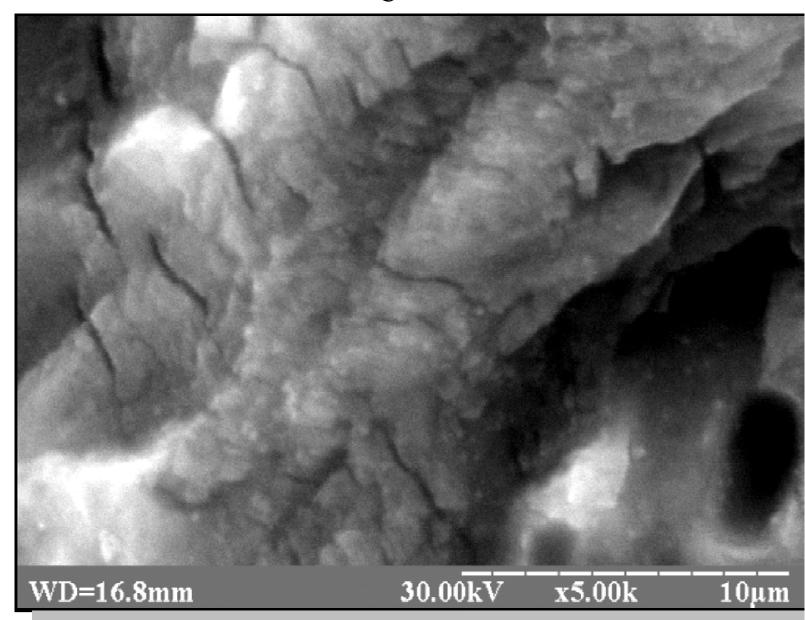

d

Fig.10 Fatigue mechanisms of crack propagation 
The last phase of macrocrack propagation is a zone of final stage of failure. On a Fig. 11, shown an area of a transition of fatigue growth of macrocrack into a ductile pit failure. However, a surface of fatigue failure is covered by facets, but they do not create brook patterns; but rather positioned chaotically. The surface of cavities and facets with clear boundaries (Fig. 11a) are well visible secondary microcracks which located in the area of cavities, on the edges of facets (Fig. 11b). Furthermore, it is possible to distinguish two types of microcracks, deep one, mentioned earlier and small one, which are located across facets edges, practically parallel one to another. Such positioning may suggest that failure is realized on inter-crystal planes by the method of breakage, practically brittle. Cracks that initiated in facets are a consequence of cyclical deformation of material during prior period.

By summarizing general analysis mechanism of micro-failure and propagation of macrocrack, right away there is a need to distinct main influence of microcracks on a structural level on whole process of failure. The next defining factor, that is unconditionally a type of load. Moreover, it possible to suppose that cyclical load with steady amplitude in general feature very adequate recreates processes that realize in real constructions. Of course, real load has random character, but in our opinion change in mechanism of failure would consist only in strengthening or leveling of some parts, and general character of failure mechanism would remain.

In the development of the models for prediction of initiation and propagation of short fatigue cracks is required to take into an account influence of microstructural changes, since as it is shown by conducted analysis processes which realized on microlevel significantly determine general streaming of macrofracture and determine general lifetime of a construction.

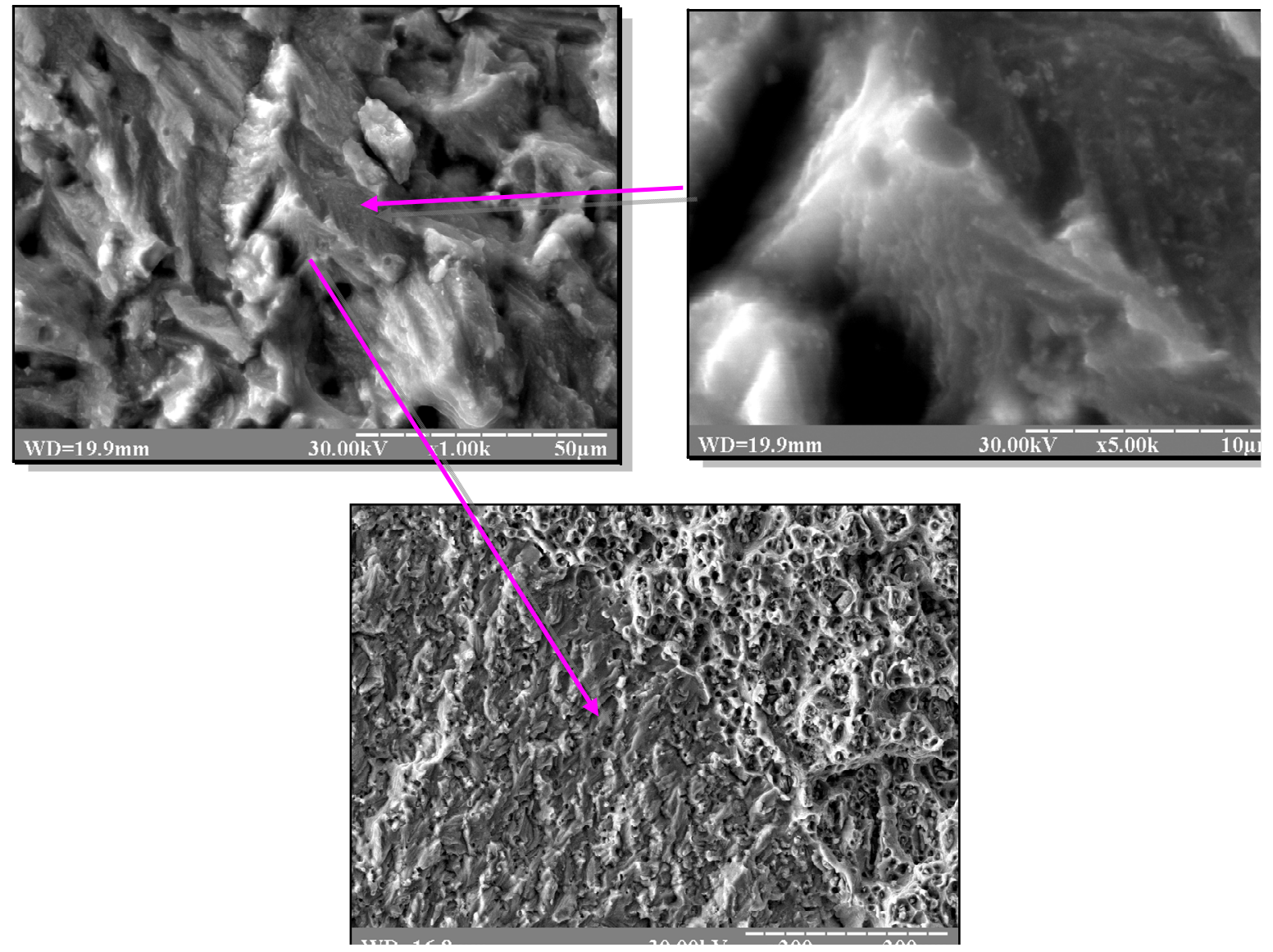

Fig.11 Fatigue mechanisms propagation crack 


\section{CONCLUSION}

1. On a basis of conducted research worked out method of determining of initial micro-damage of elements in rivet joint with help of use of vortex-current detector. There was established boundary sizes of initial fracture in a form of short crack which in this case was determine by length about $500 \mathrm{mkm}$ and width $30 \mathrm{mkm}$ that clearly defined by a device under the head of a rivet. Appearance of that crack corresponded to $5 \times 104-105$ cycles, which composed $0,2-$ 0,35 of total quantity of cycles prior to fracture of a joint or element.

2. It was analyzed micromechanism of fracture of aluminum alloy 2024-T3 in conditions of steady-amplitude cyclical load. It was established that fracture executes during realization of a complex mechanism, which includes brittle and plastic part, which overlay on whole netting system of microstructural cracks. This collaboration is realized differently on different areas of propagation of fracture macrocrack.

3. The influence of microstructural changes must be considered during design of any model for prediction of crack propagation, like on the stage of short and also on the stage of long crack, especially when predicting or estimating resource remainder. Since, as seen from presented analysis processes which occur on the microlevel in generally determine streaming of macrofracture and also determine general lifetime of the construction.

\section{REFERENCES}

[1] Li, X. D., \& Edwards, L. (1995). Analysis of short crack growth from microscopic fatigue properties. Teoretical and Applied Fracture Mechanics, 23(3), 187-198.

[2] Pedro M. G. P. Moreira, Paulo F. P. de Mates \& Paulo M. S. T. de Castro. (2005). Fatigue striation spacing and equivalent initial flaw size in Al 2024-T3 riveted specimens. Teoretical and Applied Fracture Mechanics, 43(1), 89-99.

[3] Kocanda, D., Hutsaylyuk, V., \& Hlado, V. (2007). Analize propagation short fatigue crack from hole and micromechanism fracture lamer sheet aluminium alloy 2024-T3. Military University of Technology WAT Bulletin, 56(4), 37-54.

[4] Kocanda, D., Kocanda, S., \& Kulec, P. (2005). Short fatigue crack in 2024-T3 aerospace aluminum alloy sheet. Scientific Papers. Mechanical. Technical University of Opole, 83(2), 83-90. 